\title{
Influence of the substrate orientation on the electronic and optical properties of InAs/GaAs quantum dots
}

\author{
V. Mlinar ${ }^{\text {a) }}$ and F. M. Peeters ${ }^{\text {b) }}$ \\ Departement Fysica, Universiteit Antwerpen, Groenenborgerlaan 171, B-2020 Antwerpen, Belgium
}

(Received 3 August 2006; accepted 21 November 2006; published online 27 December 2006)

\begin{abstract}
Using three-dimensional $\mathbf{k} \cdot \mathbf{p}$ calculation including strain and piezoelectricity the authors predict the variation of electronic and optical properties of InAs/GaAs quantum dots (QDs) with the substrate orientation. The QD transition energies are obtained for high index substrates, $[11 k]$ where $k$ $=1,2,3$, and are compared with [001]. They find that the QD size in the growth direction determines the degree of influence of the substrate orientation: the flatter the dots, the larger the difference from the reference [001] case. (C) 2006 American Institute of Physics. [DOI: 10.1063/1.2424435]
\end{abstract}

Semiconductor quantum dots' (QDs) unique features make them promising candidates for various semiconductor devices. ${ }^{1}$ In the widely used Stranski-Krastanow growth mode, growth conditions determine the electronic and optical properties of QDs and also introduce size, shape, or chemical composition uncertainty. ${ }^{2}$ So far, most experimental ${ }^{3}$ and theoretical $^{4-7}$ studies were performed on QDs grown on [001] substrates.

Recently, interest has moved towards QDs grown on high index surfaces where a substantial amount of experimental work has been done. ${ }^{8-11}$ Growth of QDs on high index planes has several practical advantages. For example, growth on a [113]B substrate leads to good quality QD structures with high densities and low size dispersion which are useful for QD based lasers. ${ }^{12}$ Very recently it was found that planar and vertical ordering in QD lattices can be controlled by substrate orientation enabling three-dimensional (3D) growth ranging from a chainlike pattern to a squarelike lattice of QDs. ${ }^{13}$ From a physics point of view, different substrate orientations result in different planar projections of conduction and valence bands of the constituent crystals forming QDs. As a consequence, the photoluminescence energy is expected to change with the substrate orientation. It is of fundamental importance to understand the underlying physical features of such systems. How does the strain distribution in and around QDs depend on substrate orientations? How are the electronic structure and transition energies influenced by the substrate orientation? The aim of this letter is to answer these questions and to point out the main differences with the well investigated [001] grown QDs.

The influence of the substrate orientation is more pronounced if the degree of lattice mismatch between the dot and the barrier is larger as it is the case for InAs/GaAs QDs, where the lattice mismatch is $\sim 7.2 \%$. We consider such InAs/GaAs QDs grown on $[11 k]$ substrates, where $k$ $=1,2,3$. We tested various dot shapes and sizes. Here we present the results for two different dot shapes, lens and truncated pyramid, and three different sizes. For lens shaped QDs [see Fig. 1(a)]: $R=6.78 \mathrm{~nm}$ and $h=2.83 \mathrm{~nm}$ (L1), $R$ $=9.9 \mathrm{~nm}$ and $h=3.84 \mathrm{~nm}$ (L2), and $R=10.17 \mathrm{~nm}$ and $h$ $=10.17 \mathrm{~nm}$ (L3), and for truncated pyramid [see Fig. 1(b)]:

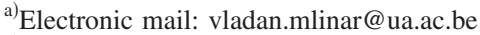

${ }^{b)}$ Electronic mail: francois.peeters@ua.ac.be
}

$b=14.7 \mathrm{~nm}$ and $h=3.4 \mathrm{~nm}(\mathrm{P} 1), b=18 \mathrm{~nm}$ and $h=3.6 \mathrm{~nm}$ (P2), and $b=22 \mathrm{~nm}$ and $h=4.5 \mathrm{~nm}$ (P3).

In our 3D model, the strain distribution of the InAs/GaAs QDs is calculated using continuum elasticity and single particle states are obtained from eight-band $\mathbf{k} \cdot \mathbf{p}$ theory $^{14}$ including strain and piezoelectricity. In order to properly take into account the effect of the different substrate orientations, the coordinate system is rotated in a way that the Cartesian coordinate $z^{\prime}$ coincides with the growth direction [Figs. 1(a) and 1(b)]. ${ }^{15}$ The general $[11 k]$ coordinate system $\left(x^{\prime}, y^{\prime}, z^{\prime}\right)$ is related to the conventional [001] system $(x, y, z)$ through a transformation matrix $U=U(\phi, \theta)$. The angles $\phi$ and $\theta$ represent the azimuthal and polar angles, respectively, of the $[11 k]$ direction relative to the [001] coordinate system. Recently, there was a single attempt to model InAs/InP QDs grown on [113] substrate orientation using eight-band $\mathbf{k} \cdot \mathbf{p}$ model, where the employed Hamiltonian was related to [001] coordinate system and the structure was defined in the [113] direction. ${ }^{16}$ This approach did not allow us to trace the underlying physical features of the system.

For strain distribution in order to determine the character of the strain for dots grown on $[11 k]$ substrates, we decompose the calculated strain tensor into an isotropic part $\operatorname{Tr}(e)=e_{x x}+e_{y y}+e_{z z}$ and a biaxial part $B$ $=\sqrt{\left(e_{x x}-e_{y y}\right)^{2}+\left(e_{y y}-e_{z z}\right)^{2}+\left(e_{z z}-e_{x x}\right)^{2}}$, where $e_{\alpha \beta}$ denotes the $\alpha \beta$ component of the strain tensor. The strain profiles along the growth direction across the lens shaped L3 and truncated pyramidal P1 QDs are shown in Figs. 1(c) and 1(d), respectively. First, for [001] grown QDs, the isotropic strain is negative (compressive) inside the dot and tends to zero rapidly in the barrier. This isotropic strain is increased in $[11 k]$ grown flat QDs regardless of the dot shape, and the largest increase was found for [111] grown dots. However, this is no longer the case for larger dots (pyramidal, half-spherical, or conusoidal QDs), where variation of the substrate orientation keeps the isotropic strain almost constant in the growth direction of the dot. Second, for [001] grown QDs the biaxial strain is nonzero inside the dot and reaches a significant amount into the barrier decaying very slowly to zero. For flat dots the biaxial strain is almost constant inside the dot [Fig. $1(\mathrm{~d})$ ], while for the larger dots it has a distinct minimum in the QD [Fig. 1(c)]. QD growth on [11k] surfaces does not modify the qualitative behavior of biaxial strain but just decreases the biaxial component regardless of the dot size and 
(a)
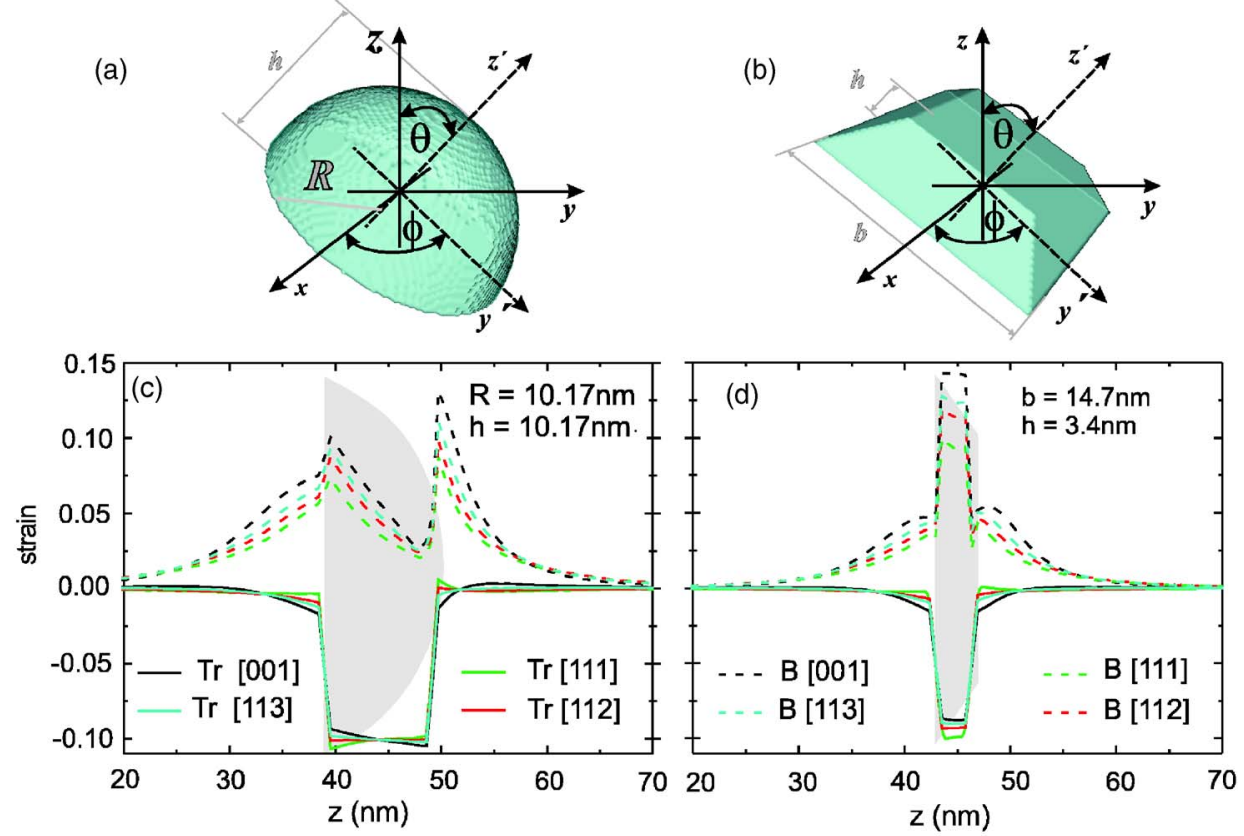

(b)

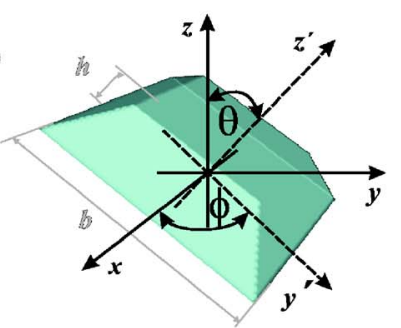

(b)

FIG. 1. (Color online) For lens shaped (a) and truncated pyramidal (b) QDs, relationship of the general $[11 k]$ coordinate system to the conventional [001] coordinate system $[\phi=\pi / 4, \theta$ $=\arctan (\sqrt{2} / k)]$. Isotropic $\operatorname{Tr}$ (solid lines) and biaxial part $B$ (dashed lines) of the strain tensor for lens shaped (c) and truncated pyramidal (d) QDs. The gray surfaces in (c) and (d) represent the dots in the growth direction. (e) $e_{x z}$ strain component of L3 QD in the plane demonstrating an increase of the shear strain with changing substrate orientation.

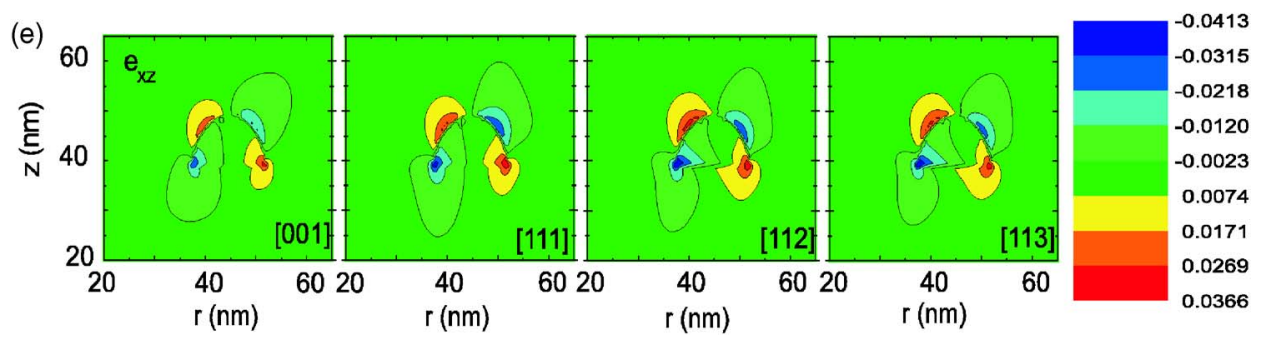

shape. Third, for all the considered dot sizes and shapes shear strains are increased for $[11 k]$ growth. As an example, we show in Fig. 1(e), for the L3 QD, the $e_{x z}$ strain component as it varies with substrate orientation. These shear components lead to a strongly asymmetric piezoelectric potential for $[11 k]$ grown QDs, influencing the distribution of electron and hole wave function inside the dot.

For electronic structure using a diagonalization of the eight-band Hamiltonian, including the strain and the piezoelectric potential, confined electron and hole energy levels are obtained numerically, which are shown in Fig. 2. In the upper panels of Figs. 2(a) and 2(b), the lowest lying electron energy levels of lens shaped and truncated pyramidal QDs are shown. Variation of the electron energy levels with the substrate orientation depends on the dot size in the growth direction and is mainly influenced by the hydrostatic strain. For smaller dots the influence of the substrate orientation on the electron energy levels is larger. The situation with the hole states is more complex. In the lower panels of Figs. 2(a) and 2(b) the hole energy levels are shown. First, one should know that for $[11 k]$ substrate orientation, different valence bands interact even at the zone center, preventing us from classifying the hole states as a heavy hole or light hole. Such a simplified, but useful picture was widely used in studies of [001] grown QDs, although, strictly speaking, this heavyhole, light-hole classification even for [001] grown QDs is valid only at the zone center. Next, the origin of the variation of hole energy levels with the substrate orientation can be traced back to the competition of several effects: (i) hydrostatic component of the strain tensor responsible for the shift of the valence bands downwards, (ii) biaxial component of Downloaded 03 Jan 2007 to 143.129.131.61. Redistribution subject

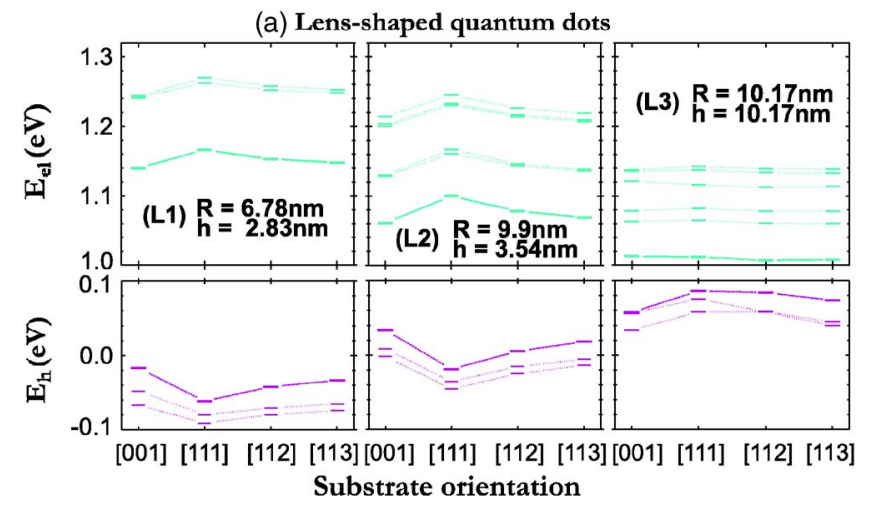

(b) Truncated-pyramidal quantum dots

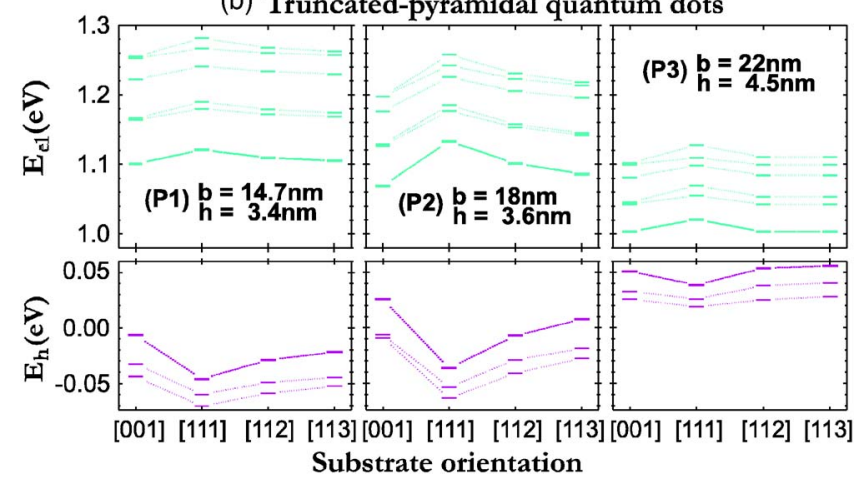

FIG. 2. (Color online) Electron and hole energy levels as they vary with the substrate orientation for lens shaped (a) and truncated pyramidal (b) QDs. Electron and hole energies are given with respect to the top of the valence band of InAs.

to AIP license or copyright, see http://apl.aip.org/apl/copyright.jsp 


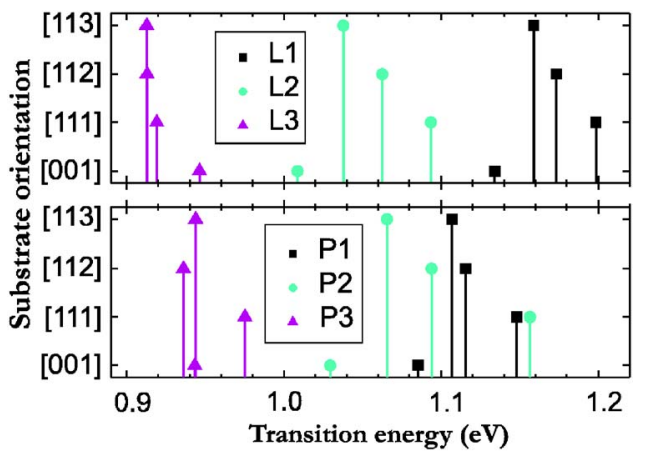

FIG. 3. (Color online) Transition energies as they vary with the substrate orientation for lens shaped (upper panel) and truncated pyramidal (lower panel) QDs.

the strain tensor influencing the degree of the valence band mixing, and (iii) variation of the hole effective mass with the substrate orientation, since it can significantly alter the effects of the size quantization in QD. The hole effective mass along the given momentum direction can be obtained by determining the energy dispersion relation from the kinetic part of the Hamiltonian, and for the bulk case, it is largest for the [111] surface. As a result of competition of the above three effects, we can distinguish two opposite cases as a function of QD size in the growth direction: (i) flat dots, where the variation of hole energy levels with the substrate orientation is mainly influenced by the hydrostatic strain, and (ii) large dots [see Fig. 2, L3 QD], where the band mixing resulting from the kinetic part of the Hamiltonian, and reduced biaxial part of the strain tensor, has a dominant influence on the variation of the hole levels with substrate orientation. We stress that the shape of the dot does not influence the above conclusions.

For transition energies including direct Coulomb interaction in our calculations, the variation of the transition energy with the substrate orientation and QD size and shape is shown in Fig. 3. For flat dots, the transition energies of [11k] grown QDs are increased with respect to those of the reference [001] grown QDs. The largest difference from the reference case exhibits [111] grown QD, whereas [113] grown QDs have transition energies that are closest to the ones of [001]. The situation with the larger dots is opposite, where the transition energies of [11k] grown large QDs are smaller as compared to [001] grown QDs. These findings are a direct consequence of previous discussion for single particle states. An intermediate case is illustrated by the example of P3 QD, where the transition energies of [112] and [113] grown QDs are lower than the one of the [001] grown QD, but the tran- sition energy of the [111] grown QD is higher.

In conclusion, our $3 \mathrm{D} \mathbf{k} \cdot \mathbf{p}$ calculation predicts the dependence of the transition energies of InAs/GaAs QDs on substrate orientation. We show that the QD size in the growth direction determines the degree of the influence of the substrate orientation on the electronic and optical properties of [11k] grown QDs, whereas the influence of the shape is of secondary importance. The flatter the dots are, the larger the difference from the reference [001] case. Although composition intermixing and shape variation related to the growth conditions can quantitatively influence our results, the presented work should be understood as a guideline for the variation of the electronic and optical properties of QDs going from the well investigated [001] grown QDs to [11k] grown QDs, where $k=1,2,3$.

This work was supported by the Belgian Science Policy (IUAP) and the European Union Network of Excellence: SANDiE.

${ }^{1}$ V. Shchukin, N. N. Ledentsov, and D. Bimberg, Epitaxy of Nanostructures, Nanoscience, and Technology (Springer, New York, 2003), Chap. 5, pp. 315-335.

${ }^{2}$ V. Shchukin and D. Bimberg, Rev. Mod. Phys. 71, 1125 (1999).

${ }^{3}$ P. W. Fry, I. E. Itskevich, D. J. Mowbray, M. S. Skolnick, J. J. Finley, J. A. Barker, E. P. O'Reilly, L. R. Wilson, I. A. Larkin, P. A. Maksym, M. Hopkinson, M. Al-Khafaji, J. P. R. David, A. G. Cullis, G. Hill, and J. C. Clark, Phys. Rev. Lett. 84, 733 (2000).

${ }^{4}$ L. He, G. Bester, and A. Zunger, Phys. Rev. Lett. 94, 016801 (2005).

${ }^{5}$ O. Stier, M. Grundmann, and D. Bimberg, Phys. Rev. B 74, 081305 (1999).

${ }^{6}$ W. Sheng and J.-P. Leburton, Phys. Rev. B 63, 161301 (2001).

${ }^{7}$ V. Mlinar, M. Tadić, and F. M. Peeters, Phys. Rev. B 73, 235336 (2006).

${ }^{8}$ Z. Gong, Z. C. Niu, and Z. D. Fang, Nanotechnology 17, 1140 (2006); P. M. Lytvyn, I. V. Prokopenko, V. V. Strelchuk, Y. I. Mazur, Z. M. Wang, and G. J. Salamo, J. Cryst. Growth 284, 47 (2005).

${ }^{9}$ S. Godefroo, J. Maes, M. Hayne, V. V. Moshchalkov, M. Henini, F. Pulizzi, A. Patane, and L. Eaves, J. Appl. Phys. 96, 2535 (2004).

${ }^{10}$ Y. Temko, T. Suzuki, and K. Jacobi, Appl. Phys. Lett. 82, 2142 (2003); 83, 3680 (2003).

${ }^{11}$ M. C. Xu, Y. Temko, T. Suzuki, and K. Jacobi, Phys. Rev. B 71, 075314 (2005).

${ }^{12}$ P. Caroff, C. Paranhoen, C. Platz, H. Folliot, N. Bertru, C. Labbe, R. Piron, E. Homeyer, A. Le Corre, and S. Loualiche, Appl. Phys. Lett. 87, 243107 (2005).

${ }^{13}$ M. Schmidbauer, Sh. Seydmohamadi, D. Grigoriev, Zh. M. Wang, Yu. I. Mazur, P. Schäfer, M. Hanke, R. Köhler, and G. J. Salamo, Phys. Rev. Lett. 96, 066108 (2006).

${ }^{14}$ V. Mlinar, M. Tadić, B. Partoens, and F. M. Peeters, Phys. Rev. B 71, 205305 (2005).

${ }^{15}$ R. H. Henderson and E. Towe, J. Appl. Phys. 78, 2447 (1995); G. A. Baraff and D. Gershoni, Phys. Rev. B 43, 4011 (1991).

${ }^{16}$ C. Cornet, A. Schliwa, J. Even, F. Doré, C. Celebi, A. Létoublon, E. Macé, C. Paranthoën, A. Simon, P. M. Koenraad, N. Bertru, D. Bimberg, and S. Loualiche, Phys. Rev. B 74, 035312 (2006). 